\title{
Ubiquitous Health Care Monitoring Services (UHCMS): Review of Opportunities and Challenges
}

\author{
Norah I. Alsaeed \\ Department of Information Systems, \\ King Khalid University \\ Abha, Saudi Arabia
}

\author{
Nouf S. Aldahwan \\ Computing and Information Technology \\ King Abdulaziz University \\ Jeddah, Saudi Arabia
}

\begin{abstract}
Expansion in government spending on healthcare that is driven by an increasing population, limited economic and human capital plus the rise in the prevalence of chronic diseases are seen as the causes for the growing pressure on health care institutions. As technology advances, health care providers are now shifting toward ubiquitous health care systems. Since, hospitals can decrease the daily pressure by moving the simple medical tasks and regular checks to the home which is called Ubiquitous Health Care Monitoring System (UHCMS). This study aims to review UHCMS literature for the last two years to understand the researches' contributions and shortages in this field and discuss the literature gap for further investigations.
\end{abstract}

\section{Keywords}

Ubiquitous computing, Ubiquitous health care, health care monitoring systems, IoT-based health care services.

\section{INTRODUCTION}

The progress of information technology is radically changing the live, specially what is called Ubiquitous computing paradigm, enables the design of embedded devices with sensing, analyzing, and communication abilities along with other equipment to understand and aware regarding their environment. Nowadays, rising trend in health care is to shift regular medical assessments and other health care facilities from hospital to the home setting which is called Ubiquitous Health Care Monitoring System (UHCMS) [1].

Consequently, Hospitals can decrease the daily pressure by moving the simple medical tasks to the home. Therefore, it is essential that emerging technologies are introduced in the health sector to develop automated healthcare techniques and to utilize them to monitor patients from anywhere and at any time. Many solutions are proposed for adopting different technologies in order to enable UHCMS, these technologies allow tracking and processing of different health care matrices accurately, and securely in real time [1].

Researchers interested to health care services arena struggle with unreliable data, slow communications, and gaping health

workflows generated by health care providers and incompatible care services, that makes difficult to provide efficient home monitoring care systems. A major issue is to enable a reliable sensing, analysis and communicating model that can link these independent health care services together to create an open end-to-end network.

This research tends to answer the following question; what are the researches' contributions and limitations in the context of ubiquitous health care monitoring systems from 2018 to
2020? In order to answer the question, the research tries to achieve the following objectives,

- To define the UHCMS and its characteristics

- To explain the architecture of UHCMS from different point of views

- To analyze UHCMS solutions through their contributions and limitations based on the literature.

- To discuss the UHCMS assessment issues which addressed by the reviewed solutions.

The rest of the report is organized as follows: Section II introduces a background about UHCMS, its definitions, characteristics, architecture from multiple perspectives, advantages, and challenges. Section III. presents the research methodology. Section IV. presents research result (i.e. UHCMS survey). Section V. discusses the reviewed papers. Last section concludes the paper and presents future work.

\section{BACKGROUND}

UHCMS is not only a simple technology improvement, it is also, multilevel and all-around changes, UHCMS definition and related characteristics, architectural dimensions, advantages, challenges are presented as follows:

\subsection{UHCMS Definition}

UHCMS is a method of delivery of healthcare services which uses ubiquitous computing platforms to track the health of patients outside the traditional healthcare setting. UHCMS extends to the different technologies used to transfer information electronically between doctors and patients, and it is various distribution mechanisms within the wider telehealth market.

\subsection{UHCMS Characteristics}

The characteristics of UHCMS can be divided into three categories: First, applications-oriented frameworks need to assure robust transmission between sensors and smartphone, create a customized network between the sensors and the computer device, and protect the patient`s information.

Second, things-oriented frameworks must be applicationbased flexible, real-time tracking, on-time distribution, high sensitivity, achieve higher performance at lower power dispersion, and focus on highly intelligent handling.

Last category, semantic oriented frameworks should be able to establish patterns of behavior rely on the knowledge previously learned, utilize language natural processing (NLP) mechanisms to enhance experience of user, and possess pervasive computational capabilities. 
Other relevant characteristics involve heterogeneous processing, simultaneous communication throughout all nodes of the network, context aware processing, adaptive networks that can handle a vast number of computers as necessary, and higher-efficiency resource computing.

\subsection{UHCMS Architecture}

Health care monitoring services from high level point of view concerns with the continuous tracking of significant signs (rate of heart, temperature, pressure of blood, and etc.) to detect anomalies. If an anomaly is detected, a healthcare provider shall provide effective health advice or/and intervention. This ultimately affects the healthcare workflow and involves a change from a doctor centered to a patient centered approach as illustrated in figure 1 [3].

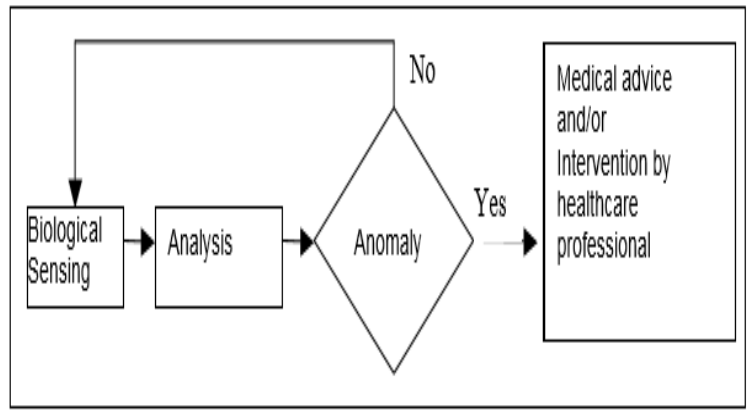

Figure 1: UHCMS Architecture [3]

Architecture of UHCMS can be viewed from three main perspectives: Configuration, Framework, and Organization:

1. UHCMS configuration refers to the installation in suitable settings of various physical components that could be arranged to resolve core problems. Heterogeneous processing solutions are designed to use these systems in integrated healthcare processing environments by placing the appropriate sensors and actuators into environments.

2. UHCMS framework contains the libraries and contexts such that health care services architectures can be operate. Healthcare framework can be broadly divided into three frameworks: networking, computing, and service.

3. UHCMS organization architecture arranges the requirements of the physical components of healthcare in the design hierarchy.

4. Ubiquitous healthcare architecture requires interoperability through various technologies. For example, a personal network (PAN) will communicate with the embedded sensors [2].

\subsection{UHCMS Advantages}

There are many benefits of UHCMS [4], some of them are highlighted as follows:

- Accessibility of knowledge and expertise of health care field, Access includes knowledge of the respective member's health situation, thus knowledge might include current situation of the patient, potential history of diseases, treatments, operations, current indications or diagnosis currently available.

- Quality health care services on a high equipped basis to served urban and rural areas.
- UHCMS facilities are required for new and complementary (noninvasive) health procedures.

- Cost reductions in administrative, transportation, and claim management expenses for UHCMS services providers and patients.

- Use of traditional emergency services is reduced.

- Non-emergency infrastructure enhanced.

- Time reduced for non-emergency services.

- Critic's immediate accessibility.

\subsection{UHCMS Challenges}

UHCMS faces major challenges need to be addressed by researchers interested in the field of ubiquitous health care services [3], this section discusses some of them and the related parameters:

- Reliability: Factors influencing infrastructure reliability and solutions based on ad hoc wireless devices include coverage of network, the prevalence of slowdowns, services range, available energy, protocol of routing, computer or network failure, and other devices those have uncooperative behaviors.

- Power Management: Trade-off exists between the heavy equipment and the amount of battery recharging. The key factors influencing power utilization include the size and frequency of the information being transmitted and the routing mechanisms used for information transmission.

- Scalability: The health care monitoring systems need to scale up and down in regarding the number of patients being monitored and can be supported reliably. T Bit rate, tracking and transmission frequency and the amount of information distributed for each patient are the factors affecting the scalability.

- Security and privacy: Privacy means a patient's right to limit the dissemination and acquisition of their personal data. Whereas security defines as the protection of health information of each patient from unintended access. Security and privacy are key barriers to the largescale deployment and adoption of ubiquitous, wearable deviceenabled computing.

- Invisibility: The systems that facilitate pervasive observation should be merged into the context so that patients would conduct their everyday activities without becoming aware about sensors and devices embedded.

\section{METHODLOGY}

This section describes the process of performing the review; the followed steps were:

1. Identifying the keywords such that most of the research papers involved in proposing the UHCMS model. The suggested keywords entered for this work were ubiquitous health care monitoring systems, IoT-based monitoring health care systems, and pervasive health care monitoring services.

2. Searching the papers from electronic databases, involving; Elsevier Science direct, IEEE, Springer Link, and Google scholar. The search was conducted for papers published from 2018 to 2020 in order to review 
latest studies in the interested area by using the identified search keywords.

3. A content analysis has been performed on all identified papers. All papers were assessed by papers titles, then by papers abstracts and for most relevant papers, the researcher access and read the papers full text.

The literature about ubiquitous health care monitoring systems has been collected and reviewed based on qualitative content analysis methodology.

\section{UHCMS REVIEW}

The mentioned process revealed about 50 papers focused on UHCMS as the main research purpose during the last two years. This paper selected 12 papers those most relevant and provide details about their proposed systems for further analysis and deep understanding of researches' contributions and limitations. Table 1 summarizes the significant reviewed papers.

Ubiquitous health care monitoring systems depends highly on the biosensors' devices accuracy for improving the remote diagnosis and treatment efficiency [5]. Therefore, it is important for such systems to provide real time notifications through high performance IoT devices and Wide Body Area Network (WBAN) [6]-[8]. The proposed model incorporates a three-way approach to data collection verification: First, vital signs are captured in the first step by remote medical devices and eventually transmitted to the base computer. Then, the assigned nurse conducted physical readings of the same parameters or factors manually through using standard strategies. At the end, every estimate produced by the medicinal devices was physically documented by the analyst to check any loss of remote transmission data, incorrect transmission of information and delay of transmission.

The general framework of the proposed diagnostic and monitoring system uses tablets, offered a secured accessible gateway that allows the information network and links it to the individual patient profile, including the points of interest of the devices (serial Low. and address). The details that must be accessed at the end of the clinicians by using a special user name and hidden key. Because of the possibility of outside access to the touchy recorded data; the system actively ignores any opened device available. Mobile 3G information was used in this way via the protected firewall and switch configurations associated with the tablets and smart phones.

Most of the proposed solutions depend upon techniques of machine learning such as classification, clustering and edge computing technology [9]-[11]. First, a set of information (arrows which are thick) is imported onto the system. The required data are obtained by choosing the area of analysis (DB of GIS and DB of Personal data). Processing of the imported data is then carried out, extreme data is observed and missing one is expected. In the reasoning phase, the primary information is inferred from the low-level details. It is interesting that some qualitative data are retained in the archive directly and the other are collected through the process of reasoning (arrows which are thin). The standard patient's expiratory flow values (PEF) are different for each person with in term of age, height, and sex. The personal data is then used to reason the typical PEF parameters. Patient asthmatic cases are expected. After that, it defines suitable areas for each patient. Finally, monitoring of asthma patients is performed depending on the expected expiratory peak flow (PEF) parameters results.

For autonomous diagnosis and treatment is most likely to utilize knowledge-based systems for reasoning and recommendations [12][13]. The blockchain aware (BBAware) design has three main components: a remote application as a server along with web-services for communications, a mobile application and a smart watch software. The smart watch framework has the Complex Sense Acquisition element, in which the patient obtains latest time and rate of heart. Locally within a log archive, this data remains until the computer gets connections to the Internet to sync up data with the web-services. There are two key modules in the mobile application. The Context-Acquisition module collects profile data from the doctor and the other module is Notifier that Notifies doctor, family members, or the patient by adaptation software after evaluating the patient's heartrate and when patient specifically says it doesn't feel well. The remote server provides web-services for the synchronization of data between mobile app and smart watch. In addition, the retrieval of the context data is performed by a Reasoner module. The remote server also has a history archive, a Data Processing, and a knowledge-based features.

The security of sensitive patients' information is a vital issue for UHCMS, many solutions adopt block chain structure for enabling secured and distributed transmission of Notifications, however, such solutions fail to decrease the time required for data transmission which has high priority for monitoring systems [14]. A patient who is monitored remotely is fitted with multiple medical equipment, such as a pump of insulin or monitor of blood-pressure. The raw data is transferred by the software to a master "modular synthesizer" for aggregation and structuring. Upon the completion, the structured data is sent for full analysis to the appropriate smart-contract along with the threshold which is customized. The source of the data fed to the smart-contracts is defined in "Oracle" through the Ethereum platform. In this scenario, the Oracle is the smart agent that specifically interacts with the smart-contracts. The smart-contract will then analyze the data supplied and provide warnings to the patient and provider of healthcare services, along with automated medication instructions for the nodes of actuators if it is required.

On the other hand, using fuzzing algorithms and other artificial intelligent solutions empower the secure data transmission as well as they decrease the time required for transmission [15][16].

Table 1 Summary of Significant Literature

\begin{tabular}{|c|c|c|c|}
\hline Ref. & Purpose & Contribution & Limitation \\
\hline [9] & $\begin{array}{l}\text { Ube-Health framework for pervasive } \\
\text { healthcare services, which utilizes, } \\
\text { high-performance computing, deep } \\
\text { learning, big data, the Internet of } \\
\text { Things (IoT), and edge computing in } \\
\text { order to support UHCS challenges. }\end{array}$ & $\begin{array}{l}\text { The first research that discusses thoroughly } \\
\text { the numerous network-related issues in the } \\
\text { next generation healthcare systems, using } \\
\text { advanced data mining and deep learning } \\
\text { techniques to improve quality of services } \\
\text { parameters. }\end{array}$ & $\begin{array}{l}\text { The study does Not consider the } \\
\text { privacy, security, scalability, and } \\
\text { reliability of the ubiquitous healthcare } \\
\text { systems. }\end{array}$ \\
\hline
\end{tabular}




\begin{tabular}{|c|c|c|c|}
\hline [10] & $\begin{array}{l}\text { The proposed system implements an } \\
\text { intelligent wearable data capture } \\
\text { system with specific identification } \\
\text { mechanisms which require simple } \\
\text { classification based on thresholds and } \\
\text { less processing time. }\end{array}$ & $\begin{array}{l}\text { This design decreases the delay and leads } \\
\text { the Healthcare Information System (HIS) to } \\
\text { be closed to optimized meaning. }\end{array}$ & $\begin{array}{l}\text { The complex policies are produced on } \\
\text { fly and addressed when the devastating } \\
\text { issue is resolved. This dynamic } \\
\text { configuration can result in costly } \\
\text { malfunctions and complexities. }\end{array}$ \\
\hline [11] & $\begin{array}{l}\text { A framework for monitoring out-door } \\
\text { asthma depends on Geographic } \\
\text { Information System (GIS) in the } \\
\text { context of pervasive systems. }\end{array}$ & $\begin{array}{l}\text { The feasibility of the proposed } \\
\text { framework is evaluated by applying it to an } \\
\text { actual data sample consisting of internal } \\
\text { contextual information involving personal } \\
\text { data of patients, gender, age medical, } \\
\text { history height), locations of patients and the } \\
\text { peak of patient's expiratory flow values. }\end{array}$ & $\begin{array}{c}\text { Shortage of accessing all asthma } \\
\text { factors which are risky, such as the } \\
\text { locations of allergens for the asthmatic } \\
\text { patient. }\end{array}$ \\
\hline [12] & $\begin{array}{l}\text { Reasoning of ontology model based } \\
\text { on rules that provide a context aware } \\
\text { system for patients with COPDs. }\end{array}$ & $\begin{array}{l}\text { The ontology-based framework can offer a } \\
\text { more accurate way of handling medical } \\
\text { data. Additionally, the introduction of an } \\
\text { SWRL layer of rules for experts on top of } \\
\text { OWL could manage various kinds of } \\
\text { context and offer accurate } \\
\text { recommendations. }\end{array}$ & $\begin{array}{l}\text { The proposed system was Not tested } \\
\text { for evaluating the perceptions of } \\
\text { patients and testing their effectiveness } \\
\text { and efficiency through actual } \\
\text { implementation and crossing trials. }\end{array}$ \\
\hline [13] & $\begin{array}{c}\text { BB-Aware (Beta-Blocked-Aware) } \\
\text { project adopts a ubiquitous approach } \\
\text { that applies the principles of } \\
\text { pervasive healthcare to support } \\
\text { patients through beta-blocked } \\
\text { therapies. }\end{array}$ & $\begin{array}{l}\text { The study provides integrated research } \\
\text { approach for the monitoring of patients } \\
\text { being treated with beta-blocking } \\
\text { medications. }\end{array}$ & $\begin{array}{l}\text { The study did Not provide an actual } \\
\text { experiment of the proposed } \\
\text { architecture for real patients; therefore, } \\
\text { the architecture is still not evaluated. } \\
\text { The study did Not use intelligent } \\
\text { approach such that using some of the } \\
\text { machine learning techniques to } \\
\text { identify the degree of risk for smart } \\
\text { Notifications instead of regular } \\
\text { Notifications or beside each other. }\end{array}$ \\
\hline [14] & $\begin{array}{l}\text { A private block chain through the } \\
\text { Ethereum standard in which the } \\
\text { sensors interact with a smart } \\
\text { system that allows smart contracts } \\
\text { and tracks all events on the block } \\
\text { chain. }\end{array}$ & $\begin{array}{l}\text { Blockchain has the ability to enhance } \\
\text { security in the patient monitoring } \\
\text { platforms and to simplify the distribution of } \\
\text { Notifications related to health on the basis } \\
\text { of standards compliance. }\end{array}$ & $\begin{array}{l}\text { The study fails to achieve less timing } \\
\text { for transmission. Decisions need to be } \\
\text { taken on one patient basis at a time, } \\
\text { depending on the nature of the } \\
\text { condition and on the type of sensor. }\end{array}$ \\
\hline [15] & $\begin{array}{l}\text { Activity tracking and recognition } \\
\text { relying on a multiple class } \\
\text { cooperation of classification process } \\
\text { to increase the accuracy of } \\
\text { classification in fog-supporting } \\
\text { videos through block chain } \\
\text { architecture. }\end{array}$ & $\begin{array}{l}\text { Fog computing not only eliminates the } \\
\text { delay in uploading large volumes of streams } \\
\text { to cloud computing servers but rather } \\
\text { decreases Internet bandwidth usage and } \\
\text { related financial costs. }\end{array}$ & $\begin{array}{l}\text { The complexity of the system and } \\
\text { power consuming along with the high } \\
\text { cost. }\end{array}$ \\
\hline [16] & $\begin{array}{l}\text { A fuzzy algorithm and neural- } \\
\text { networks, into a secured health care } \\
\text { monitoring framework to allow the } \\
\text { system to act as an intelligent health } \\
\text { care platform, which decides on its } \\
\text { own priority based on the parameters } \\
\text { of health collected from sensors. }\end{array}$ & $\begin{array}{c}\text { The study develops a reliable flexible } \\
\text { authentication framework securing } \\
\text { patients health data and ensuring secure } \\
\text { interaction. }\end{array}$ & $\begin{array}{l}\text { The proposed solution does not } \\
\text { provide a mechanism for handling the } \\
\text { exponential scalability of the system. }\end{array}$ \\
\hline
\end{tabular}

The model comprises of a trusted setting which is responsible for handling authenticated biomedical information from the body of the patient, then it transmitted to the Hub of IoT Microsoft Azure via GSM modem where original data is transformed into semantic representation, using a logic algorithm that is learned in a fuzzy inference-based system (FBIS) to obtain the patient's status. The proposed framework offers accurate, reliable, real-time and secure monitoring for patients through integrating FBIS with a secure monitoring system to obtain the patient's condition and submit it to the initial precautions medical advisory.

\section{DISCUSSION}

As a result of papers analysis, the UHCMS proposed solutions can be assessed through their abilities to address UHCMS issues; they are reliability, cost effective, scalability, diagnosis and treatment mechanism, time required for transmission of data, security, integrity, and energy efficiency. The proposed models which adopts fog and cloud computing success to provide a high level of scalability and time response [15]. On 
the other hand, the solutions which uses machine learning and reasoning systems provide autonomous diagnosis and treatment. The block chain technology ensures high security and integrity of such services.

The vision is to provide a comprehensive system where specific patient health information are regularly captured and examined for anomalies and a warning is sent to a control center to provide suitable guidance or actions by health care providers. Many of the techniques already exist to make the vision a reality. However, there are a number of issues to be considered while working on ubiquitous monitoring framework to be a realistic solution.

\section{CONCLUSION}

In fact, there is no other sector in which the value of obtaining the correct information at the correct time, regardless of time and distance, is critical more than the health care. Therefore, ubiquitous computing offers significant opportunities for health care monitoring services. However, the obstacles of designing, deploying and managing such services are emerged due to need for more accurate, cost effective, secure, real time, and power efficient systems. This paper surveys most recent UHCMS proposed solutions and discusses their contributions and limitations in term of the existent challenges. Future work concerns with proposing comprehensive UHCMS model which attempts to improve the current solutions.

\section{REFERENCES}

[1] S. Mohapatra, S. Mohanty, and S. Mohanty, "Smart healthcare: an approach for ubiquitous healthcare management using IoT," Big Data Analytics for Intelligent Healthcare Management, Academic Press, 2019, pp. 175-196.

[2] "Smart Healthcare using IoT-Aware Architecture for Health Monitoring," in International Journal of Recent Technology and Engineering, vol. 8, no. 5, pp. 5620$5624,2020$.

[3] K. Panagiotis and I. Georgia, "Ubiquitous Patient Monitoring," in Journal on Information Technology in Healthcare, vol.7, pp.202-213, 2009.

[4] Z. Omary, F. Mtenzi, B. Wu and C. O'Driscoll, "Ubiquitous Healthcare Information System: Assessment of its Impacts to Patient's Information", in International Journal for Information Security Research, vol. 1, no. 3, pp. 71-77, 2011.

[5] S. Kim, S. Yeom, O. Kwon, D. Shin and D. Shin, "Ubiquitous Healthcare System for Analysis of Chronic Patients' Biological and Lifelog Data," in IEEE Access, vol. 6, pp. 8909-8915, 2018.

[6] W. D. Patel, S. Pandya, B. Koyuncu, B. Ramani, S. Bhaskar, and H. Ghayvat, "NXTGeUH: LoRaWAN based NEXT Generation Ubiquitous Healthcare System for Vital Signs Monitoring \& Falls Detection," 2018 IEEE Punecon, IEEE, 2019, pp. 1-8.
[7] O. Geman, I. Chiuchisan, I. Ungurean, M. Hagan, and M. Arif, "Ubiquitous healthcare system based on the sensors network and android internet of things gateway," 2018 IEEE SmartWorld, Ubiquitous Intelligence \& Computing, Advanced \& Trusted Computing, Scalable Computing \& Communications, Cloud \& Big Data Computing, Internet of People and Smart City Innovation, IEEE, 2018, pp.1390-1395.

[8] S. M. Ghatole, "Ubiquitous Healthcare System and Wireless Biosensors," in International Journal of Multidisciplinary Educational Research, vol.9, no.2, 2020.

[9] T. Muhammed, R. Mehmood, A. Albeshri and I. Katib, "UbeHealth: A Personalized Ubiquitous Cloud and EdgeEnabled Networked Healthcare System for Smart Cities", IEEE Access, vol. 6, pp. 32258-32285, 2018.

[10] U. Qidwai, J. Chaudhry, S. Jabbar, H. Zeeshan, N. Janjua and S. Khalid, "Using casual reasoning for anomaly detection among ECG live data streams in ubiquitous healthcare monitoring systems", Journal of Ambient Intelligence and Humanized Computing, vol. 10, no. 10, pp. 4085-4097, 2018. Available: 10.1007/s12652-018-1091-x.

[11] N. Kaffash-Charandabi, A. Alesheikh and M. Sharif, "A ubiquitous asthma monitoring framework based on ambient air pollutants and individuals' contexts", Environmental Science and Pollution Research, vol. 26, no. 8, pp. 7525-7539, 2019.

[12] H. Ajami, H. Mcheick, K. Mustapha, "Ubiquitous Healthcare Systems and Medical Rules in COPD Domain," International Conference on Smart Homes and Health Telematics, Springer, Cham..2019 ,108-97.pp

[13] A. V. D. da Silva, and V. Vieira, "BBAware-A ContextAware Mobile and Wearable Architecture for Monitoring Beta-Blocked Cardiac Patients," in Anais do VIII Simpósio Brasileiro de Computação Ubiqua e Pervasiva, SBC, 2020, pp. 31-39.

[14] K. Griggs, O. Ossipova, C. Kohlios, A. Baccarini, E. Howson and T. Hayajneh, "Healthcare Blockchain System Using Smart Contracts for Secure Automated Remote Patient Monitoring", Journal of Medical Systems, vol. 42, no. 7, 2018.

[15] N. Islam, Y. Faheem, I. Din, M. Talha, M. Guizani and M. Khalil, "A blockchain-based fog computing framework for activity recognition as an application to eHealthcare services", Future Generation Computer Systems, vol. 100, pp. 569-578, 2019.

[16] H. El Zouka and M. Hosni, "Secure IoT communications for smart healthcare monitoring system", Internet of Things, p. 100036, 2019. 